\title{
Conocimiento teórico y apego al procedimiento de diálisis peritoneal del paciente o su familiar
}

\author{
Wendy Juana María García Meza ${ }^{1}$, Ana Laura Carrillo Cervantes², María de los Ángeles Villarreal Reyna ${ }^{3}$, \\ María Magdalena Delabra Salinas²
}

${ }^{1}$ Licenciada en enfermería. ${ }^{2}$ Maestra en ciencias de enfermería. ${ }^{3}$ Doctora en ciencias de enfermería, docente investigadora de la Universidad Autónoma de Coahuila. México

\section{Resumen}

Introducción: Paciente y familiar deben conocer los conceptos esenciales y poseer habilidades motoras para la realización de diálisis peritoneal continua ambulatoria, por esto, enfermería brinda educación al paciente y familiar sobre los cuidados de diálisis en el hogar, sin embargo, continúan acudiendo pacientes con alguna complicación al hospital.

Objetivo: Determinar el nivel de conocimiento teórico y apego al procedimiento dialítico del paciente o familiar.

Metodología: Se realizó un estudio descriptivo. Se efectuó una visita domiciliaria, en donde se aplicaron: una lista de cotejo para evaluar el apego al procedimiento, un cuestionario para evaluar los conocimientos teóricos y una cédula de variables clínicas y sociodemográficas. Participaron 24 sujetos, se dividieron en grupo pacientes (GP), donde los pacientes se auto-realizan el procedimiento y familiar (GF), donde los familiares realizan la técnica.

Resultados: El nivel de conocimiento teórico en general fue moderado $(73.24 \%)$, el GP obtuvo un índice más elevado $(75.35 \%)$ que el GF $(71.45 \%)$. En la técnica, en general, se adhirieron al $80.3 \%$ de los pasos, el GF obtuvo mayor índice correcto (81.92\%) que el GP $(78.63 \%)$.
Conclusión: Los pacientes y familiares demuestran un déficit en ambas áreas, por esto, es necesario evaluarlos periódicamente para detectar fallos en la técnica y en el conocimiento, que predispongan al paciente a una complicación prevenible.

\section{PALABRAS CLAVE}

- DIÁLISIS

- CONOCIMIENTO Y APEGO

Theoretical knowledge and adherence to peritoneal dialysis procedure by the patient or a family member

\begin{abstract}
Introduction: Patient and family should know the essential concepts and possess motor skills for conducting continuous ambulatory peritoneal dialysis. Therefore, education of patient and family about the care of home dialysis is carried out by nurses, however, patients with complications continue going to the hospital.
\end{abstract}

Objective: Determine the level of knowledge and adherence to the dialysis procedure by the patient or a family member.

Methods: A descriptive study was conducted. In a home visit the following instruments were applied: a checklist to assess adherence to procedure, a questionnaire to assess the theoretical knowledge and a questionnaire of clinical and sociodemographic variables. They involved 24 subjects. They were divided into patient group (PG), 
where patients self-perform the procedure and family group $(F G)$, where family carried out the technique.

Results: The level of knowledge was generally moderate (73.24\%), the $P G$ obtained a higher rate $(75.35 \%)$ than the FG $(71.45 \%)$. In general, on the technical knowledge there was an adherence of the $80.3 \%$ to the steps, the FG obtained higher correct index (81.92\%) than the PG $(78.63 \%)$.

Conclusion: Patients and families show a deficit in both areas, so it is necessary to evaluate them periodically to detect failures in technique and knowledge that predispose the patient to a preventable complication.

\section{KEYWORDS}

- DYALISIS

- KNOWLEDGE AND ATTACHMENT

\section{Introducción}

La Insuficiencia Renal Crónica (IRC) es un padecimiento que exige alto apego al tratamiento; el cual, se encuentra influido por el conocimiento que posee el individuo responsable ${ }^{1}$. Según datos del Instituto Nacional de Estadística y Geografía, para el 2012, la IRC se situó como la onceava causa de muerte en México con 11,955 defunciones $^{2}$. Esta enfermedad es consecuencia de los resultados deficientes en el tratamiento de la hipertensión arterial y la diabetes mellitus ${ }^{3,4}$, ésta última constituye más del $50 \%$ de los casos de pacientes renales que inician algún tratamiento renal sustitutivo ${ }^{4}$.

Uno de los tratamientos renales sustitutivos es la Diálisis Peritoneal Continua Ambulatoria (DPCA), que es un procedimiento realizado por el paciente o su familiar en el domicilio después de una capacitación proporcionada por enfermería ${ }^{5}$, puesto que el paciente y familiar serán el equipo de salud en el hogar, deben conocer los conceptos de la enfermedad y poseer habilidades motoras para la realización de la técni$\mathrm{Ca}^{6,7}$, con lo que se pretende que éstos controlen y/o resuelvan cuando sea posible y sobre todo informen a la unidad cuando aparezcan problemas que afectan al cuidado integral del paciente ${ }^{8,9}$.
Aunque la diálisis peritoneal ha incrementado la sobrevida de los pacientes; a menudo se asocia con peritonitis, que es la complicación de mayor prevalencia, que impacta en la calidad de vida, supervivencia, estado nutricional, funcionalidad y viabilidad del tratamiento ${ }^{3,4}$. Y que puede asociarse con la falla en la técnica de $\mathrm{DPCA}^{10}$, por olvido del conocimiento y/u omisión o modificación del procedimiento ${ }^{11}$; por ello una de las metas de los profesionales de enfermería es conseguir que pacientes y familia tengan un nivel adecuado de conocimientos sobre su enfermedad y cuidados $^{12}$; por esto debe mantenerse el contacto, seguimiento y evaluación éstos para poder resolver dudas y reforzar conocimientos, para incidir en la disminución de complicaciones por olvidos o mala práctica.

En la literatura, los resultados obtenidos son controversiales, algunos autores reportan que los pacientes en DPCA poseen un nivel de conocimientos teóricos adecuados $^{13}$ y aplican la técnica de forma correcta ${ }^{10,}$ 13; mientras que otros sostienen un promedio bajo en la asimilación del conocimiento y en la habilidad para efectuar el procedimiento ${ }^{14}$. Asimismo, señalan defectos en la técnica, como el empleo de jabón no antiséptico para el lavado de manos $^{15,16}$; familiares realizan los cambios de bolsa sin entrenamiento y el roce de la terminal con la mesa ${ }^{17}$. Así como, la falta de los registros correspondientes, de inspección del líquido drenado y de la bolsa y comprobación de la bolsa antes de realizar el procedimiento ${ }^{15,16}$. Añadiendo que la prevalencia de peritonitis se relaciona con el no uso de mascarilla ${ }^{16}$.

Por lo tanto, se debería realizar un seguimiento, mediante la aplicación de evaluaciones posteriores a la capacitación para determinar si el paciente o el familiar responsable realizan la DPCA conforme al procedimiento establecido evitando complicaciones. Asimismo, brindar retroalimentación, que reforzará el aprendizaje, afianzará lo aprendido y modificará comportamientos viciados ${ }^{6}$.

Por lo tanto, el objetivo de la presente investigación es determinar el nivel de conocimiento teórico y apego al procedimiento dialítico del paciente o familiar.

\section{Material y método}

Se efectuó un estudio descriptivo transversal, se empleó un censo de los sujetos inmersos en un programa de DPCA (24 participantes) de un hospital de tercer nivel en Saltillo, Coahuila, México. Para la colecta de datos se llevó a cabo una visita al domicilio de cada 
paciente en tiempos de recambio de solución. Los participantes se dividieron en dos grupos: el grupo paciente ( $G P: f=11$ ) donde los pacientes se auto-realizaban la DPCA y el grupo familiar ( $G F: f=13$ ) donde los familiares realizaban el procedimiento.

Se incluyeron a los pacientes y familiares que realizan el procedimiento de DPCA con bolsa gemela Ultra Bag Baxter ${ }^{\circledR}$, que no se encontraran en capacitación, que el paciente no estuviera internado en la institución y que aceptaron participar mediante la firma del consentimiento informado según el Reglamento General de Salud en Materia de Investigación de la Secretaría de Salud y la Declaración de Helsinki. El nivel de riesgo es mínimo, ya que no se realizó ninguna intervención para modificar comportamientos de ninguna índole.

Se aplicó una cédula de variables sociodemográficas, una cédula de variables clínicas y del procedimiento, un cuestionario de 38 reactivos de opción múltiple y relación elaborado en exprofeso, para medir el conocimiento teórico, basado en la Guía de inducción al paciente en diálsis peritoneal (18) y valorado por expertos de la unidad de Diálisis, contenía 4 dimensiones: conocimientos generales, dieta, medicamentos y configuración del sistema Ultra Bag Baxter ${ }^{\circledR}$ de Bolsa Gemela; se evaluó mediante índices $0-100 \%$, se establecieron cuartiles donde $<$ de $70=$ escaso, de $71-80 \%=$ moderado, de $81-90 \%=$ sustancial y de $91-$ $100 \%=$ extenso; $y$ una lista de cotejo para valorar el procedimiento de cambio de solución de Diálisis con bolsa gemela Ultra $\mathrm{Bag}^{\circledR}$ de Baxter; el paso de aplicación de medicamentos se evaluó aparte de la lista de cotejo, ya que no todos los participantes aplicaban medicamento en la bolsa de solución, resultando un total de 20 pasos. Los pasos eran marcados como correctos solamente si eran completa y correctamente realizados. Los datos se analizaron por medio del programa estadístico SPSS V19, empleando estadística descriptiva.

\section{Resultados}

De los 24 participantes que conformaban la población, se tomaron dos grupos: el grupo de pacientes (GP) conformado por $41.4 \%(f=11)$ y grupo de familiares (GF) por $58.6 \%(f=13)$. Del GP, la mayoría fueron hombres $(63.6 \%), 72.7 \%$ casados, $45.5 \%$ se dedicaban al hogar; $45.5 \%$ contaban con estudios medios superiores (Tabla 1), tenían un promedio de 55 (DE \pm 9.79) años de edad, 17.09 ( $D E \pm 12.25$ ) días capaci-

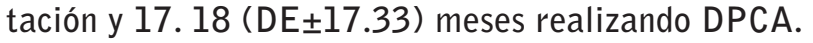

Tabla 1. Variables sociodemográficas.

\begin{tabular}{|c|c|c|c|c|}
\hline Sexo & $\begin{array}{c}\text { GP } \\
f\end{array}$ & $\%$ & $\begin{array}{c}\mathrm{GF} \\
F\end{array}$ & $\%$ \\
\hline Femenino & 4 & 36.4 & 9 & 69.2 \\
\hline Masculino & 7 & 63.6 & 4 & 30.8 \\
\hline \multicolumn{5}{|l|}{ Estado civil } \\
\hline Soltero & - & - & 1 & 7.7 \\
\hline Casado & 8 & 72.7 & 11 & 84.6 \\
\hline Unión libre & 1 & 9.1 & - & - \\
\hline Divorciada & 2 & 18.2 & 1 & 7.7 \\
\hline \multicolumn{5}{|l|}{ Ocupación } \\
\hline Hogar & 5 & 45.5 & 8 & 61.5 \\
\hline Trabajo & 2 & 18.2 & 4 & 30.8 \\
\hline Pensionado & 1 & 9.1 & - & - \\
\hline Jubilado & 1 & 9.1 & 1 & 7.7 \\
\hline Incapacitado & 2 & 18.2 & - & - \\
\hline \multicolumn{5}{|l|}{ Parentesco } \\
\hline Independiente & 11 & 100 & - & - \\
\hline Esposo (a) & - & - & 8 & 61.5 \\
\hline Hijo (a) & - & - & 4 & 30.8 \\
\hline Otros familiares (cuñado) & - & - & 1 & 7.7 \\
\hline \multicolumn{5}{|l|}{ Escolaridad } \\
\hline Analfabeta & 1 & 9.1 & 1 & 7.7 \\
\hline Sabe leer y escribir & 1 & 9.1 & - & - \\
\hline Estudios básicos & 4 & 36.4 & 6 & 46.2 \\
\hline Estudios medios o superiores & 5 & 45.5 & 6 & 46.2 \\
\hline
\end{tabular}

FUENTE: Cédula de datos sociodemográficos (elaboración por los autores) $\mathrm{N}=24$.

Como enfermedades predominantes se encuentran la diabetes mellitus $(63.6 \%)$ e hipertensión arterial (100\%). Tres pacientes $(27.3 \%)$ han presentado episodios de peritonitis. Asimismo, 7.7\% realizaba DPCA sin estar capacitado. El $36.4 \%$ se pesa con la frecuencia requerida; $45.5 \%$ mide frecuentemente la presión arterial, $45.5 \%$ emplea jabón antibacterial para el lavado de manos, el $54.5 \%$ restante emplea jabón neutro, de tocador o gel, $90.9 \%$ conserva sus uñas limpias y cortas y $36.4 \%$ señala tener algún problema visual. El $90.9 \%$ realiza la DPCA en dormitorio y $9.1 \%$ en la sala. El $90.9 \%$ presenta edema corporal, $81.8 \%$ en miembros inferiores, $36.4 \%$ tiene algún signo de deterioro de la integridad cutánea (enrojecimiento, secreción y costra) en el orifico de salida del catéter. El $36.4 \%$ pesa el líquido drenado en báscula; $18.2 \%$ emite a su juicio un aproximado, $36.4 \%$ mide en recipien- 
tes graduados y $9.1 \%$ no cuantifica y solo $18.2 \%$ cuantifica correctamente ingresos y egresos.

En el GF, 69.2\% eran mujeres, $84.6 \%$ casados, $61.5 \%$ se dedicaba al hogar, $46.2 \%$ estudios superiores, $61.5 \%$ eran los cónyuges del paciente, tenían un promedio de $52.62(D E \pm 12.19)$ años de edad, 17.15 ( $D E \pm 8.33$ ) días de capacitación y 22.85 ( $D E$ \pm 22.08 ) meses realizando diálisis. Como enfermedades principales se encuentran la diabetes mellitus $(53.8 \%)$ e hipertensión arterial (23.1\%). Seis de los pacientes a los que dializan han presentado peritonitis $(46.2 \%)$ y tres han sido reincidentes $(23.1 \%)$, 9.1\% realizaba DPCA sin estar capacitados, $23.1 \%$ pesa al paciente con la frecuencia requerida, 38.5\% mide frecuentemente la presión arterial. Para el lavado de manos $53.9 \%$ emplea jabón antibacterial, $46.1 \%$ restante emplea jabón neutro, de tocador 0 gel y $84.6 \%$ conserva sus uñas limpias y cortas.

El 53.8\% menciona tener algún problema visual. $7.7 \%$ realiza diálisis en un cuarto especial, $76.9 \%$ en el dormitorio y $15.4 \%$ en la sala, $38.5 \%$ presenta edema corporal, $23.1 \%$ en miembros inferiores; $38.5 \%$ posee algún signo de deterioro de la integridad cutánea en el orifico de salida del catéter (enrojecimiento, secreción y costra), $61.5 \%$ pesa el líquido drenado en báscula; $15.4 \%$ emite a su juicio un aproximado, $15.4 \%$ lo mide en recipientes graduados y $7.7 \%$ no lo cuantifica; y $15.4 \%$ cuantifica correctamente ingresos y egresos.

En el conocimiento teórico, en el área de conocimientos generales, ambos grupos presentaron mayor índice de error en la identificación de síntomas de uremia (GP: $72.7 \%$; GF: 69.2\%) y signos de sobrecarga de líquidos (GP: $36.4 \%$; GF: $46.2 \%$ ). El $72.7 \%$ del GP y $76.9 \%$ del GF identifica los síntomas de infección del orificio de salida del catéter y conocen que hacer en caso de que éstos se presenten; y $63.6 \%$ del GP y $76.9 \%$ del GF conocen los síntomas de peritonitis.

El $63.6 \%$ del GP y $76.9 \%$ del GF no relaciona correctamente los alimentos con las sustancias que se deben de consumir, evitar o limitar. Los dos grupos mostraron bajo índice correcto medicamentofunción al relacionar la vitamina D (GP: $27.3 \%$; GF: $30.8 \%$ ), quelante de fósforo (GP: $45.5 \%$; GF: $38.5 \%$ ) y hierro (GP: $45.5 \%$; GF: $46.2 \%$ ). Sobre la configuración del sistema de bolsa gemela ultra bag Baxter ${ }^{\circledR}$, en ambos grupos se observa mayor error en la identificación del protector de salida (GP: $63.6 \%$; GF: $53.8 \%$ ).
La dimensión de conocimientos generales fue más afectada (promedio de acierto: GP $45.45 \%$ y GF $46.15 \%$ ), que las demás áreas (dieta: GP 72.72\% y GF 76.92\%; medicamentos: GP $65.65 \%$ y GF $66.66 \%$ : y configuración del equipo; GP 86.01 y GF 74.55 (Tabla 2).

Tabla 2. Índice promedio por dimensión del instrumento teórico.

\begin{tabular}{lcc}
\hline & $\begin{array}{c}\text { GP } \\
\text { Índice } \\
\end{array}$ & GF \\
& $\overline{\mathbf{X}}$ & $\overline{\mathbf{X}}$ \\
\hline Conocimientos generales & 45.45 & 46.15 \\
Dieta & 72.72 & 76.92 \\
Medicamentos & 65.65 & 66.66 \\
Configuración del sistema de bolsa & 86.01 & 74.55 \\
\hline
\end{tabular}

FUENTE: Instrumento teórico sobre IRCT y tratamiento (elaboración por los autores) $\mathrm{N}=24$.

En el conocimiento teórico, el índice correcto obtenido fue moderado; el GP obtuvo un promedio de $75.35 \%$ y $71.45 \%$ del GF (Tabla 3 ).

Tabla 3. Índice promedio del instrumento teórico total.

\begin{tabular}{lcc}
\hline & $\begin{array}{c}\text { Índice } \\
\text { GP }\end{array}$ & GF \\
\hline Media & 75.35 & 71.45 \\
DE \pm & 16.77 & 16.87 \\
Mínimo & 52.63 & 26.32 \\
Máximo & 97.37 & 97.37 \\
\hline
\end{tabular}

FUENTE: Instrumento teórico sobre IRCT y tratamiento (elaboración por los autores) $\mathrm{N}=24$.

Entre los errores en la técnica se encontró que: $72.7 \%$ del GP y $84.6 \%$ del GF no verifica las características completas del equipo antes de comenzar a dializar; $53.8 \%$ del GP y $81.7 \%$ del GF no realiza la limpieza de la superficie de trabajo; $9.1 \%$ del GP y $15.4 \%$ del GF emplean incorrectamente el cubreboca; $63.6 \%$ del GP y $38.5 \%$ del GF realiza erróneamente el lavado de manos o lo omite.; $46.2 \%$ del GF y $27.3 \%$ del GF no revisa que el tapón minicap contenga yodopovidona; y sólo $45.5 \%$ del GP y $46.2 \%$ del GF llevan a cabo el registro y balance de líquidos (Tabla 4). 
Tabla 4. Lista de cotejo para el procedimiento de cambio de solución de diálisis con Bolsa Gemela Ultra Bag ${ }^{\circledR}$.

\begin{tabular}{|c|c|c|c|c|}
\hline Preparar materiales & $\begin{array}{l}\text { Correcto } \\
\text { GP } \\
f\end{array}$ & $\%$ & GF & $\%$ \\
\hline $\begin{array}{l}\text { Reúna los materiales necesarios (una bolsa Gemela } \\
\text { Ultrabag® con líquido a temperatura deseada, una pinza roja, } \\
\text { un cubreboca y un tapón minicap). }\end{array}$ & 9 & 81.8 & 10 & 76.9 \\
\hline $\begin{array}{l}\text { Verificar la concentración, fecha de caducidad, no fugas, } \\
\text { puerto de medicamentos y tapón de anillo estén en su lugar. }\end{array}$ & 3 & 27.3 & 2 & 15.8 \\
\hline Colóquese el cubreboca (en nariz y boca). & 10 & 90.9 & 11 & 84.6 \\
\hline Limpie la superficie de trabajo. & 2 & 18.2 & 6 & 46.2 \\
\hline $\begin{array}{l}\text { Coloque los materiales sobre la superficie de trabajo } \\
\text { retirando la sobre-envoltura de la bolsa revisando } \\
\text { la transparencia de la solución. }\end{array}$ & 9 & 81.8 & 11 & 84.6 \\
\hline $\begin{array}{l}\text { Exponga la línea de transferencia del paciente verificando } \\
\text { que esté cerrada. }\end{array}$ & 8 & 72.7 & 13 & 100 \\
\hline $\begin{array}{l}\text { Lávese las manos con agua y jabón, séquelas perfectamente. } \\
\text { Conectar la bolsa gemela Ultra Bag® }\end{array}$ & 4 & 36.4 & 8 & 61.5 \\
\hline $\begin{array}{l}\text { Retire el tapón minicap y el tapón de anillo, conecte } \\
\text { sin contaminar las partes estériles. } \\
\text { Purgar el sistema }\end{array}$ & 11 & 100 & 12 & 92.3 \\
\hline $\begin{array}{l}\text { Rompa la cánula o frangible y deje que el líquido dialisante } \\
\text { arrastre hasta que pase la última burbuja a la bolsa de drene } \\
\text { y coloque la pinza roja en la línea de drenaje. } \\
\text { Desconectar y colocar el tapón minicap }\end{array}$ & 10 & 90.9 & 11 & 84.6 \\
\hline Lávese las manos con agua y jabón, séquelas perfectamente. & 5 & 45.5 & 11 & 84.6 \\
\hline Abra el tapón minicap y revise que la esponja interior contenga yodopovidona. & 8 & 72.7 & 7 & 53.68 \\
\hline Registrar. & 6 & 54.5 & 7 & 53.8 \\
\hline
\end{tabular}

FUENTE: Lista de cotejo para el procedimiento de cambio de solución de diálisis con bolsa gemela Ultra Bag® modificada por los autores $\mathrm{N}=24$.

El 9.1\% del GP y $38.5 \%$ del GF aplicaban medicamento en la bolsa. De éstos, $15.4 \%$ del GF y 9.1 del GP aplicaban incorrectamente los medicamentos: $9.1 \%$ del GP y $7.7 \%$ GF bañaba con yodopovidona la aguja con la que aplicaba medicamento a la bolsa para reusarla, y $7.7 \%$ del GF al aplicar medicamento a la bolsa quitaba el puerto de medicamentos y lo sumergía en alcohol. De los 24 participantes sólo $7.7 \%$ del GF se adhiere totalmente al procedimiento. Se obtuvo un promedio de apego al procedimiento de $78.63 \%$ del GP y $81.92 \%$ del GF.

\section{Discusión}

El nivel de conocimientos teóricos que poseen los pacientes y/o familiares es moderado, ya que mantienen un índice de conocimiento de $75.35 \%$ y $71.45 \%$ respectivo de cada grupo (73.4\% población en general). Manifiestan bajo apego al procedimiento, ya que demostraron cum- plir con el $78.63 \%$ y $81.92 \%$ de la técnica de DPCA ( $80.41 \%$ población en general), tal porcentaje es más elevado que el $64 \%$ reportado por González-Zamudio ${ }^{14}$.

Se detectaron defectos en la técnica como: empleo de jabón de tocador para el lavado de manos y falta de inspección de la bolsa y de los registros correspondientes similar a los resultados presentados por Sayed y Dong ${ }^{15,16}$; también el roce de la terminal de la entrada con la mesa y la realización DPCA por un familiar sin capacitación como lo reporta Marcos ${ }^{17}$.

Sin embargo, se encuentra discrepancia con Cidoncha ${ }^{13} y$ Carballo-Monreal10, ya que sostienen que los participantes tienen nivel de conocimiento teórico y realización de la técnica adecuados. No obstante, la similitud o contraste de resultados de esta investigación con otros estudios puede deberse a la diferencia del instrumento de valoración, promedio de edad, tiempo de realización de DPCA, 
tamaño de la muestra, tipo y clasificación de valoración (índices mas no categorías en el apego) y diferencia de equipo de diálisis empleado, ya que Carballo-Monreal 10 en su estudio empleó Fressenius Medical Care.

Esta investigación se encuentra limitada por el número reducido de participantes (totalidad inmersa en el programa de DPCA de un hospital de tercer nivel); por esto se sugiere ampliar el tamaño muestral; así mismo, realizar análisis de las valoraciones de conocimiento y apego por rangos de edad y género.

\section{Conclusión}

El nivel de conocimiento teórico que poseen los participantes de ambos grupos en este estudio en moderado y el apego al procedimiento fue del $80.41 \%$ en la población en general y por grupo $78.63 \%$ del GP y $81.92 \%$ del GF.

Lo que permite concluir, que pacientes y familiares integrantes de un programa de DPCA, pierden conocimientos indispensables para el cuidado del mismo paciente renal en el hogar. Por esto, se sugiere que enfermería efectúe una supervisión subsecuente que englobe valoraciones teóricas y prácticas mediante una visita domiciliaria, la cual proporcionará información real de la situación en la que se encuentra inmerso el paciente y su familiar, lo que brindará a enfermería la identificación de situaciones potenciales que puedan estar influyendo en el cuidado y que no puedan ser detectadas cuando el paciente asiste al hospital, previniendo complicaciones por olvido u omisión, tomando en consideración las características reales en las que se desenvuelve la persona en casa. También se recomienda que enfermería realice una retroalimentación de los puntos débiles observados en la visita domiciliaria, enfatizando en la realización correcta del lavado de manos, empleo de agua y jabón anti-bacterial y no uso de soluciones alcoholadas, correcto uso del cubre-boca, verificación de características del equipo, higiene del lugar de trabajo, purgado indispensable de la solución, importancia de la omisión de recambios y de la realización del balance de líquidos, ya que este paso imprescindible para identificar sobrecarga de líquidos o deshidratación.

Para finalizar, se sugiere que el personal de enfermería unifique criterios al capacitar y evaluar a pacientes 0 familiares sobre el cuidado en el domicilio, lo que permitiría estandarizar el entrenamiento en DPCA y posteriormente realizar investigaciones científicas donde las valoraciones sean similares, que permitan emitir comparaciones entre los resultados.
Recibido: 20 enero 2015

Revisado: 3 febrero 2015

Modificado: 25 febrero 2015

Aceptado: 28 febrero 2015

\section{Bibliografía}

1. Guerra VT, Díaz AE, Vidal K. La educación como estrategia para mejorar la adherencia de los pacientes en terapia dialítica. Revista Cubana Enfermería [revista en Internet]. 2010 Jun [consultado 2015 Mar 11]; 26 (2): Disponible en: http://scielo. sld.cu/scielo.php?script=sci_arttext\&pid=S086403192010000200007\&lng=es

2. Instituto Nacional de Geografía y Estadística. Causas de defunción de defunciones generales totales por principales causas de mortalidad, 2012 [portal en internet] 2014 febrero [acceso marzo 2014]. Disponible en http:// www3.inegi.org. $\mathrm{m} x / \mathrm{sistemas} / \mathrm{sisept} / \mathrm{Default}$. aspx $? \mathrm{t}=$ mdemol07 \&s=est $\& \mathrm{c}=23587$

3. Ávila-Saldivar MN, Conchillos-Olivares G, RojasBáez IC, Ordoñez-Cruz AE, Ramírez-Flores HJ. Enfermedad renal crónica: causa y prevalencia en la población del Hospital General La Perla. Medicina interna de México [Revista en internet] 2013 Septiembre-0ctubre [consultado 2014 septiembre 10]; 29 (5): 473-478. Disponible en http://www. medigraphic.com/pdfs/medintmex/mim-2013/mim135e.pdf

4. Martínez FA. Incidencia de peritonitis en una cohorte de pacientes con insuficiencia renal tratados con diálisis peritoneal continua ambulatoria. Portal Maestría en Salud Pública. Universidad veracuzana [tesis de maestría en internet] 2010 febrero. [consultado 2013 mayo 30]. Disponible en http:// cdigital.uv.mx/bitstream/123456789/29817/1/ AliciaMtzFlores.pdf

5. Martín JL, Cirera F, Reina M. Formación proporcionada a los pacientes de diálisis peritoneal domiciliaria en España. Revista de la Sociedad Española de Enfermería Nefrológica [Revista en internet] 2008 enero-marzo [consultado 2013 mayo 31]; 11 (1): 13-19. Disponible en http:// scielo.isciii.es/scielo.php?script=sci_arttext\&pid $=$ S1139-13752008000100003 
6. Bernardini J, Price V, Figueiredo A. Pautas/recomendaciones de ISPD. Peritoneal Dialysis International [revista en internet], 2006 noviembre [consultado 2013 junio 4]; 26 (6): 625-632. Disponible en http://www.nefro.cl/phocadownload/Registrados/guias_clinicas/ISPD\%20Guidelines $\% 20$ en $\% 20 \% 20$ entrenamiento $\% 20 \mathrm{de} \% 20$ pacientes\%202006.pdf

7. Oberto DL. Programa educativo sobre cuidados a pacientes que reciben diálisis peritoneal ambulatoria continua dirigido a familiares. Revista electrónica de PortalesMedicos.com [Revista en internet]. 2009, Abril [consultado 23 de julio del 2013] Disponible en: http://www.portalesmedicos.com/publicaciones/articles/1817/1/ Programa-educativo-sobre-cuidados-a-pacientesque-reciben-dialisis-peritoneal-ambulatoria-continua-dirigido-a-familiares.html

8. Gómez C. La enfermería en la revisión del paciente de diálisis peritoneal. Revista de la Sociedad Española de Enfermería Nefrológica [Revista en internet] 2006 [consultado 2013 julio 10]; 31: http://www. revistaseden.org/files/3076_tema20.pdf

9. Gómez AC, Ojeda MA, Carballo E, Ramírez MA, Cárcamo J, Fernández D. Los indicadores del manejo del régimen terapéutico y su relación con la evolución de la información adquirida durante el entrenamiento en diálisis peritoneal. Revista de la Sociedad Española de Enfermería Nefrológica [Revista en internet] 2011 abril-junio [consultado 2013 julio 10]; 14 (2): 83-89. Disponible en http://scielo.isciii.es/scielo.php?pid=S1139$13752011000200002 \&$ script $=$ sci_arttext

10. Carballo-Monreal M, Ortega N, Lizarraga E. Apego individual y familiar al tratamiento de diálisis peritoneal ambulatoria continua. Revista de enfermería Instituto Mexicano de Seguro Social [Revista en internet]; 2008 [consultado 2013 mayo 24]; 16 (1): 13-18. Disponible en http:// www.medigraphic.com/pdfs/enfermeriaimss/eim2008/eim081d.pdf

11. Peláez $B$, Fernández $M$, Núñez $M$, González I, Méndez A, Quintana A. Evaluación de los conocimientos prácticos de los pacientes prevalentes en diálisis peritoneal. Enfermería Nefrológica [Revista en internet] 2013 julio septiembre [consultado 2014 enero 02]; 16 (3): 179-184.

12. Luengo, CE, Araneda G, López MA. Factores del cuidador familiar que influyen en el cumplimiento de los cuidados básicos del usuario postrado. Index de enfermería [Revista en internet] 2010 Marzo [consultado 2013 Julio 23]; 19 (1): 14-18. Disponible en: http://scielo.isciii.es/scielo.php?pid=S1132$12962010000100003 \&$ script=sci_arttext

13. Cidoncha MA, Baldeón MS, Barasategui M. Análisis de las necesidades educativas en diálisis peritoneal. Presentado en XXVII Congreso Nacional de la Sociedad Española de Enfermería Nefrológica; en Libro de comunicaciones [en internet] 2002 [consultado 2013 enero 12].133-138. Disponible en https://www.seden.org/publicaciones_articulodet.asp? idioma $=\& p g=$ publicaciones_revistadet.as p\&buscar $=\& i d=121 \& i d a r t i c u l 0=1641 \&$ Datapage $i d=3 \&$ intInicio $=1$

14. González-Zamudio R. Evaluación teórica-práctica del paciente en diálisis peritoneal continua. Revista de enfermería del Instituto Mexicano del Seguro Social [Revista en internet] 2001[consultado 2013 enero 12]; 9 (1): 19-22. Disponible en http://revistaenfermeria.imss.gob.mx/ index.php/vol-09-ano-2001-num-1/234-ano2001vol-09/353-evaluacion-teorica-practica-delpaciente-en-dialisis-peritoneal-continua.html

15. Sayed SA, Abu-Aisha $H$, Ahmed ME, Elamin S. Effect of the patient's knowledge on peritonitis rates in peritoneal dialysis. Peritoneal Dialysis International [Revista en internet] 2013 JulioAgosto [consultado 2013 diciembre 22]; 33 (4): 362-366. Disponible en http://www.pdiconnect. com/content/33/4/362.long

16. Dong J, Chen Y. Impact of the bag exchange procedure on risk of peritonitis. Peritoneal Dialysis International [Revista en internet] 2010 JulioAgosto [consultado 2013 diciembre 22]; 30 (4): 440-447. Disponible en http://www.pdiconnect. com/content/30/4/440.long

17. Marcos A. Visitas domiciliarias en un programa de diálisis peritoneal continua ambulatoria. Revista de la Sociedad Española de Enfermería Nefrológica [Revista de internet] 1985 [consultado 2013 enero 12]. Disponible en http://www.seden.org/publicaciones_articulodet.asp?idioma $=\& p g=$ publicaciones_revistadet.asp\&buscar $=\& i d=101 \&$ idarticulo $=959 \& \mathrm{Da}$ tapageid $=1 \&$ intInicio $=$

18. Baxter International Incorporation. Guía de inducción al paciente en diálsis peritoneal. Jiutepec, Morelos, México: Baxter. 2009. 